\title{
Zooming in to acquire micro-reaction: Application of microfluidics on soil microbiome
}

\author{
Xiongkun Zhang ${ }^{1}$, Shan $\mathrm{Wu}^{1}$, Xiaojie Sun ${ }^{1}$, Monika Mortimer ${ }^{2}$, Yichao $\mathrm{Wu}^{1}$, Ming Zhang ${ }^{1}$, Qiaoyun Huang ${ }^{1}$, \\ Peng Cai ${ }^{1, *}$ \\ 1 State Key Laboratory of Agricultural Microbiology, College of Resources and Environment, Huazhong Agricultural University, \\ Wuhan 430070, China \\ 2 Institute of Environmental and Health Sciences, College of Quality and Safety Engineering, China Jiliang University, Hangzhou 310018, \\ China
}

\section{H I G H L I G H T S}

-Basic principles of microfluidics are introduced.

-Microfluidics to study bacterial spatial distribution and functions.

-Challenges of microfluidics for soil microbiome in future.

\section{ARTICLE INFO}

Article history:

Received September 13, 2020

Revised November 13, 2020

Accepted November 23, 2020

Keywords:

Microfluidics

Soil bacteria

Biofilms

Chemotaxis

Quorum sensing

Horizontal gene transfer
GRAPHICAL ABSTRACT

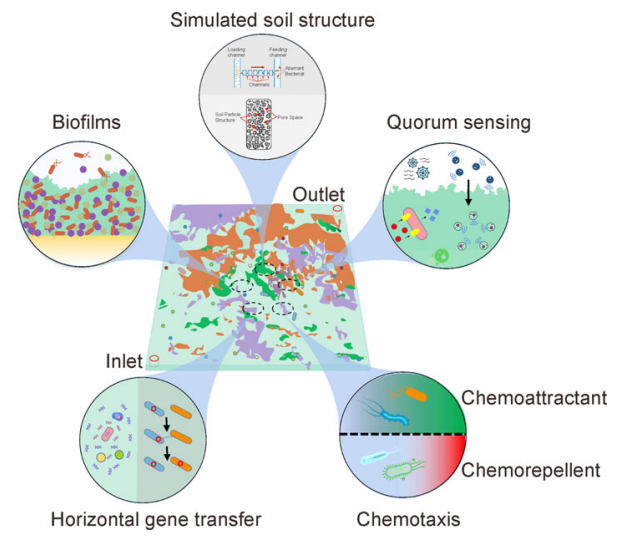

A B S T R A C T

Microfluidics confers unique advantages in microbiological studies as these devices can accurately replicate the micro- and even nano-scale structures of soil to simulate the habitats of bacteria. It not only helps us understand the spatial distribution of bacterial communities (such as biofilms), but also provides mechanistic insights into microbial behaviors including chemotaxis and horizontal gene transfer (HGT). Microfluidics provides a feasible means for real-time, in situ studies and enables indepth exploration of the mechanisms of interactions in the soil microbiome. This review aims to introduce the basic principles of microfluidic technology and summarize the recent progress in microfluidic devices to study bacterial spatial distribution and functions, as well as biological processes, such bacterial chemotaxis, biofilm streamers (BS), quorum sensing (QS), and HGT. The challenges in and future development of microfluidics for soil microbiological studies are also discussed.

(C) Higher Education Press 2021
* Corresponding author

E-mail address: cp@mail.hzau.edu.cn (P. Cai)
Bacteria are widely distributed in the soil, ocean, and atmosphere, as well as in symbiotic communities in eukaryotic organisms (Flemming and Wuertz, 2019). They play an important role in human health, soil quality and fertility, and pollution remediation, among other important areas (Stewart, 
2012; Zhu et al., 2017). Current methods of bacterial cultivation primarily include shake-flask (Koffler et al., 1945; Wu et al., 2010), 96-well plate (Miyake et al., 1992; Singh et al., 2017), and microfluidic devices. Among these cultivation methods, microfluidic devices have advantages of miniaturization, automation, and low reagent consumption. Additionally, when combined with other advanced instruments, such as the confocal laser scanning microscope, atomic force microscope, and other analytical methods (e.g., Raman spectrometry, mass spectrometry, and thermogravimetric analysis), these devices can be used to visualize and analytically characterize the dynamic processes of bacteria (Feng et al., 2015; Deng et al., 2015; Borer et al., 2018; Pousti et al., 2018). Since the beginning of the application of microfluidics in microbiology, great progress has been made in many aspects of the field, such as observing the dynamic process of biofilm formation (Feng et al., 2015; Mukherjee et al., 2016), screening soil microorganisms (Toju et al., 2018), and analyzing the effects of extracellular polymeric substances (EPS) on soil moisture (Deng et al., 2015). In recent years, manufacturing techniques like photolithography (Hassanpourfard et al., 2014) and etching (Borer et al., 2018) have allowed accurate fabrication of spatial features of bacterial habitats, including the adjustment of parameters such as volume, geometry and surface properties (Karimi et al., 2015). These fabrication techniques have enabled to create micro- or even nano-scale structures (such as micropillars) which have greatly improved the application of microfluidic technology in bacterial research.

\section{Introduction of microfluidic technology}

Microfluidics, is known as lab-on-a-chip technique. It is a miniaturized experimental platform which can be used for sample preparation, reaction, separation, and detection (Karimi et al., 2015). Microfluidics can integrate and automate multiple laboratory techniques into a system, which can be fitted on a chip up to a maximum of a few square centimeters in size. It is well-known that spatial characteristics (e.g., volume and geometry) and chemical heterogeneity of bacterial habitat play an important role in the growth and behavior of bacteria. Microfluidic devices can simulate these physical and chemical conditions associated with microorganisms allowing researchers to study the complex interactions between bacteria and their growing environment. For example, these devices can accurately mimic the environmental habitats of bacteria at the micro- or nano-scale for in-situ visualization and analysis. As shown in Fig. 1, the fluid flow can be precisely controlled by a syringe pump or constant pressure pump. The device is equivalent to a microreactor in which bacteria are cultivated and undergo various biochemical reactions (Qiu et al., 2018). While the bacterial growth dynamics can be directly observed in the device, at the same time, the effluent could be connected to an additional instrument (e.g., mass spectrometer) to perform metabolic analyses.

Currently, materials used for microfluidic chips fabrication include inorganic materials (e.g., glass), polymers (e.g.,

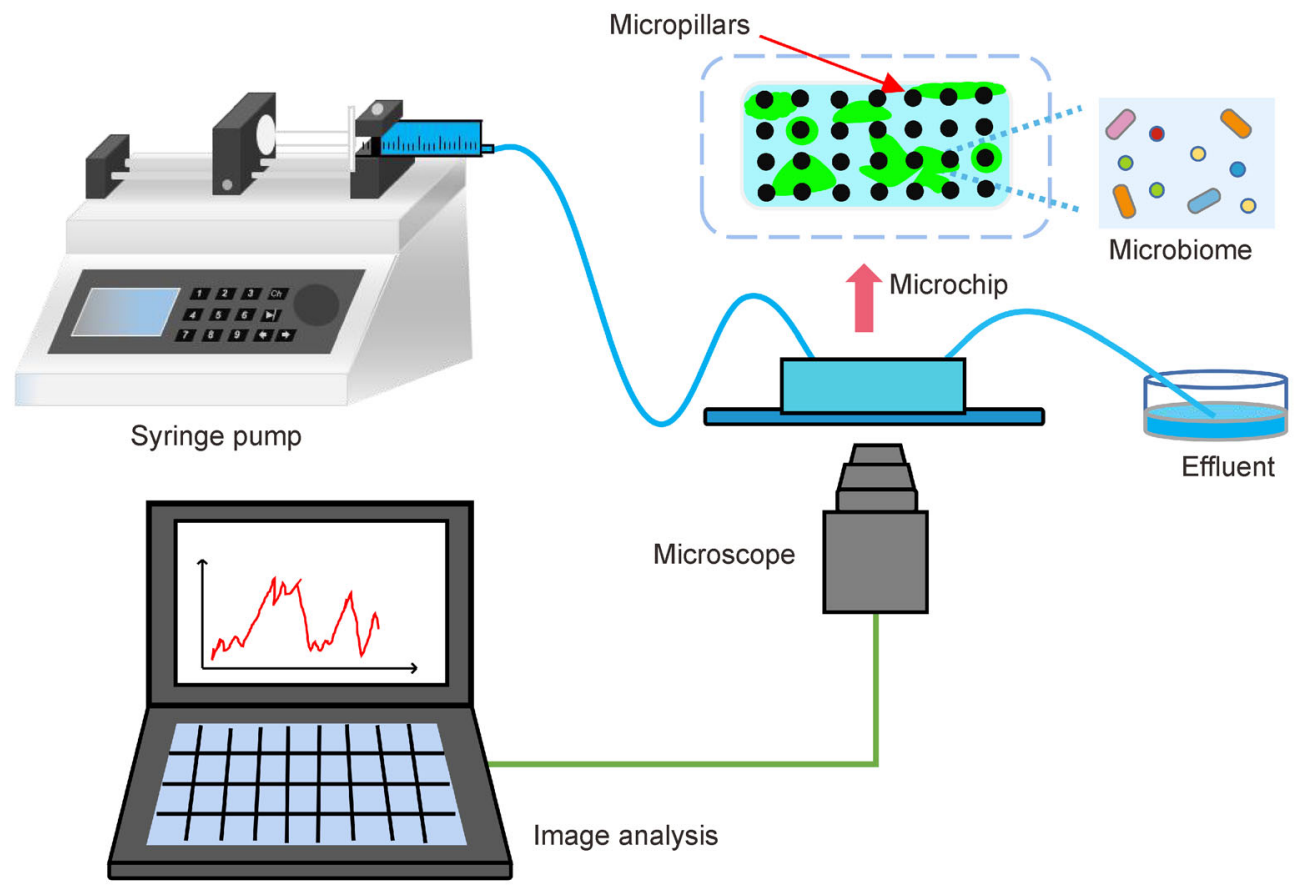

Fig. 1 The system of microfluidics. The images obtained with the microscope can be analyzed by computer. 
polydimethylsiloxane, PDMS), and paper (Karimi et al., 2015). Among them, PDMS is commonly used for microbial researches. The production of microfluidic device requires templates. To create templates, technologies such as 3D printing, glass etching, or soft lithography are often used. The accuracy of $3 \mathrm{D}$ printing and glass etching technologies is generally at the micrometer level, while the accuracy of soft lithography technology is higher and can reach the nanometer level. For example, the channels designed by Männik et al. (2009) are up to $300 \mathrm{~nm}$. After the creation of the template, PDMS is then poured on the developed template structure to fabricate the microfluidic chip. These devices have been widely used in microbial studies. For example, micropillars and porous micromodels have been used to study biofilms and simulate soil micropores as obstacles to investigate chemotaxis of bacteria (Kim et al., 2010; Singh and Olson, 2012). In addition, a "T-maze" microfluidic device has been designed to generate a concentration gradient for studying chemotaxis of bacteria (Salek et al., 2019). Also, long continuously curving structures (Drescher et al., 2013) have been used to mimic pipes in industrial or residential settings to study the shape of biofilm streamers and pipe blockages.

\section{Application of microfluidics on bacteria-environment interaction}

Soil has a complex internal environment with high heterogeneity and opacity, which limits the in situ visualization of soil microorganisms. Previous studies, which have used glass beads or artificial soil model systems to simulate the spatial structure and chemical complexity of soil or used digital image mosaics of soil thin sections to analyze microbial activities and hotspots have provided important insights into the distribution and functions of soil bacteria (Nunan et al., 2003; Gutiérrez Castorena et al., 2016; Wu et al., 2019; Zambare et al., 2019; Cai et al., 2019). However, they have not been able to simulate the spatio-temporal heterogeneity of soil at microand nanoscale. The microfluidic technology can address this problem. Microfluidics has been successfully employed in biomedicine, and the same principles can also be employed in soil science to simulate the micro-scale habitat structures (such as micropillars) and the chemically heterogeneous environment (such as signal molecules with different concentration gradients) of microorganisms to achieve in situ visualization and analysis, to understand the internal world of soil bacteria more intuitively (Aleklett et al., 2018).

Since microfluidics provides a powerful tool to control, shape, and manipulate the habitat of individual cells, it is increasingly used for studying microorganisms. In this section, we summarized the studies of bacterial spatial distribution and functions, bacterial chemotaxis, biofilm streamers, quorum sensing, and horizontal gene transfer conducted in microfluidic chips that simulate soil environments.

2.1 Simulation of soil structure to study bacterial functions

Microfluidic devices can be used to study the growth and movement of bacteria via simulation of soil pores. As shown in Fig. 2, Männik et al. (2009) designed a microchip with progressively narrower channels which connect chambers to explore the morphology of Escherichia coli and Bacillus subtilis in soil spatial structure at the submicron level. They found that both organisms could pass through the $0.4-\mu \mathrm{m}$ channel during cell division. Additionally, E. coli could pass through the channel that was less than half its own diameter, however, after passing through the channel, the morphology of the bacteria underwent various abnormal changes. The study suggested that there exists a diversity of bacterial morphologies in the submicron structure of soil. Kim et al. (2010) studied the morphology of Pseudomonas putida biofilm in a porous medium of glass microbeads. They found that the biofilm could easily detach at high flow rates, accelerating pore blockage. They also observed that under high substrate concentration the biofilm was denser and did not detach easily. Coyte et al. (2017) used microfluidic device, mechanistic models, and game theory to study the effect of porous media hydrodynamics on competition between bacterial genotypes. Their result illustrated that hydrodynamic properties profoundly affect the competition and evolution of bacteria in the porous environments. Aufrecht et al. (2019) mimicked the pore structure of sand particles on a twodimensional platform to explore the spatial evolution of Pantoea sp. YR343. It was observed that the fluid shear

A

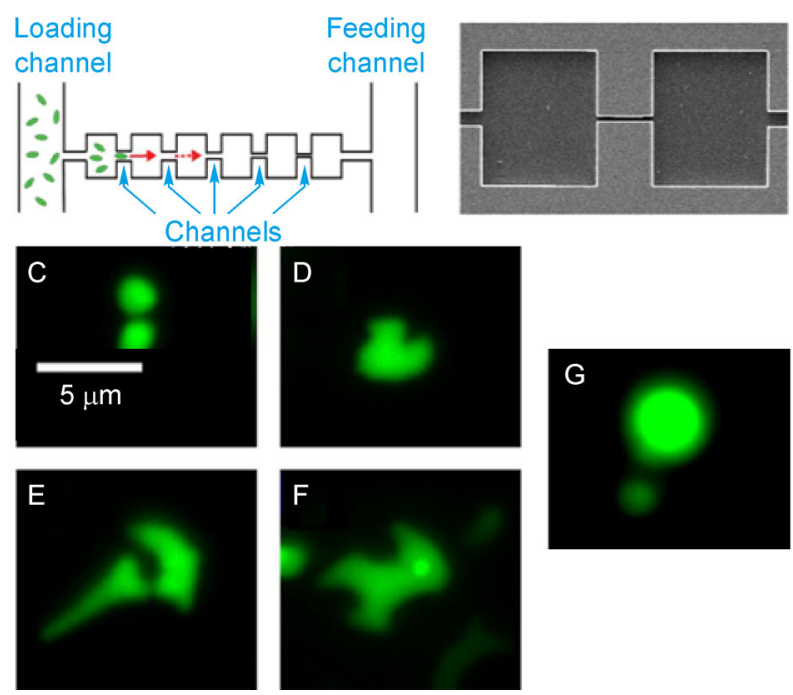

Fig. 2 Use of time-lapse fluorescence microscope for imaging bacteria in a microfluidic device. (A) Scheme of a microfluidic structure with bacteria (green) in the loading channel moving toward the feeding channel. (B) A narrow channel connects two adjacent chambers. (C-G) Fluorescence micrographs of bacteria with different aberrant shapes (Männik et al., 2009). https://doi. org/10.1073/pnas.0907542106 
force had the greatest effect on the initial spatial distribution of bacteria, however, the ability to produce EPS and biofilm expansion played a major role on the spatial distribution of bacteria over time. Huang et al. (2017) developed a SoilChip method by assembling soil suspension onto homogeneous microarray chips, which was then submerged in dissolved organic matter to initiate soil biogeochemical interfaces processes. The SoilChip combined with X-ray photoelectron spectroscopy, called SoilChip-XPS, has also been applied to study the metabolism of carbon, oxygen, nitrogen, and other elements in situ in soil-microbial complexes. Based on SoilChip, Huang et al. (2020) found that the transformation and accumulation of organic matter mediated by microorganisms preferentially attach to the existing organic-inorganic complexes. This discovery provides direct evidence for thickening nanoscale organic films at the soil micro-interfaces. Borer et al. (2018) developed an experimental platform based on glass-etched micrometric pore networks to simulate resource gradients in soil aggregates to investigate the distribution of aerobic $P$. putida and a facultative anaerobe $P$ seudomonas veronii. They found that $P$. putida accounted for $95 \%$ of the total population under the aerated conditions, while $P$. veronii accounted for $99.9 \%$ of the total population under anoxic conditions. The platform enabled direct visualization and quantification of bacterial spatial organization at the aggregate scale in pore networks.

EPS have strong water retention properties, being able to absorb amounts up to 10 times its weight (Roberson and Firestone, 1992). Thus, EPS play a significant role in improving water retention capacity of soil. Several studies have explored the effect of EPS on soil moisture in microfluidic devices. For example, Deng et al. (2015) simulated sandy loam soil to study the effects of EPS on pore water holding capacity. EPS-producing Sinorhizobium meliloti (EPS + ) and EPS-free S. meliloti (EPS-) were used in their model. The drying rate of EPS + was 1.1-2.5 times slower than that of EPS-. The experimental device allowed direct observation of the effects of EPS on water retention at soil pore scale for the first time. Guo et al. (2018) studied the effect of EPS produced by $S$. meliloti Rm1021 on moisture retention in simulated soil (1.2 $\mathrm{mm}$ macropore and sandy loam soil structure). EPS concentration had no effect on water retention in macropores, but had a strong effect on water evaporation rate and range in micropores. The water content tended to be stable at the later stage and the water retention increased with the increase of EPS concentration in micropores.

\subsection{Microfluidics in biofilm studies}

Biofilms provide numerous competitive advantages for bacteria to resist adverse external environments; these advantages include strong water retention capacity, promoting surface adhesion, and acting as a barrier against toxic substances. Biofilm streamers, a type of filamentous biofilm structure formed in porous habitats, are found widely in natural, industrial, and living environments. Normally, one end of a streamer is fixed to the surface while the other end is suspended in fluid. During the initial stage, bacteria adhere to the surface to form discrete colonies and then a biofilm. Under fluid shear, the biofilm grows linearly and a visible biofilm streamers will form over time (Valiei et al., 2012; Karimi et al., 2015).

The devices for studying biofilm streamers often have microporous or curvy structure. Marty et al. (2012) designed micropillars of different sizes and different array structures to study biofilm streamers and demonstrated that the largest streamers formed in the staggered square array of micropillars spaced $10 \mu \mathrm{m}$ apart. Valiei et al. (2012) showed that biofilm streamers of Pseudomonas fluorescens formed at a range of flow rates $\left(8,12\right.$ and $\left.20 \mu \mathrm{L} \mathrm{h}^{-1}\right)$, but the morphology of the streamers changed with the flow rate and only transient streamer formation was observed at $80 \mu \mathrm{L} \mathrm{h}{ }^{-1}$. In the vertical structure of the fluid, secondary flow played a major role in biofilm development. To better understand the role of fluidinduced stress on biofilm formation, Weaver et al. (2012) studied biofilm by using microfluidics that could generate different pressures. The biofilm biomass of Staphylococcus epidermidis 35984 (containing ica gene, i.e., able to form polysaccharide intercellular adhesin-based biofilms) increased by multiple times at $0.1-1.2 \mathrm{~Pa}$, while $\mathrm{S}$. epidermidis 12228 (lacking ica gene) could not produce biofilms at any rate. Using a microfabricated pseudo-porous platform containing a channel with micropillars, Hassanpourfard et al. (2016) demonstrated that mature biofilm streamers of $P$. fluorescens and Pseudomonas aeruginosa moved forward in the direction of flow in a nonlinear stick-slip manner. Biofilm structure remained dynamic even after clogging of the device containing micropillars. These findings have implications for design and fabrication of biomedical devices and membranetype systems, as well as understanding bacterial growth and proliferation in natural porous media such as soil and rocks. Biswas et al. (2018) visualized the development of biofilm streamers with $200 \mathrm{~nm}$ fluorescent polystyrene beads in a microfluidic device. Biofilm streamers exhibited instantaneous movement and long-term movement along the direction of the flow. There were multiple periods of stagnation and fracture during the movement process, and, eventually, the entire streamer structure would detach. Using a microporous device Scheidweiler et al. (2019) found that multi-species biofilms differentiated into basal biofilm and streamers that had similar community compositions. This highlighted the plasticity of the biofilm communities. Multi-species biofilms increased their carrying capacity and improved space utilization in porous environments (Fig. 3).

Yazdi and Ardekani (2012) placed a horseshoe-shaped structure in a micro-chamber and applied radio frequency signal to generate a pair of vortices at the air-liquid interface. $E$. coli accumulated in the vicinity of these vortices in seconds, and formed biofilm streamers in minutes. The results provided an example for developing disposable and portable microfluidic devices to be used in microbiological tests and as environmental diagnostic chips. Drescher et al. (2013) studied 
A

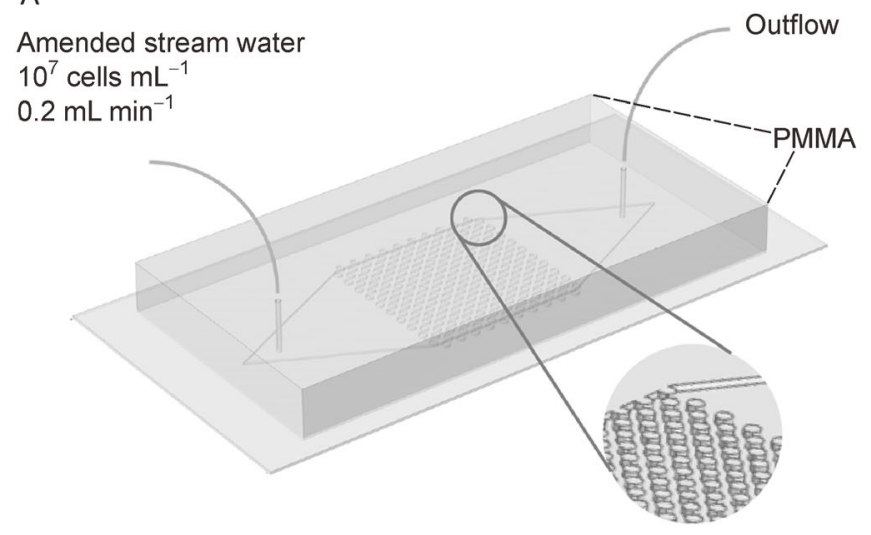

C

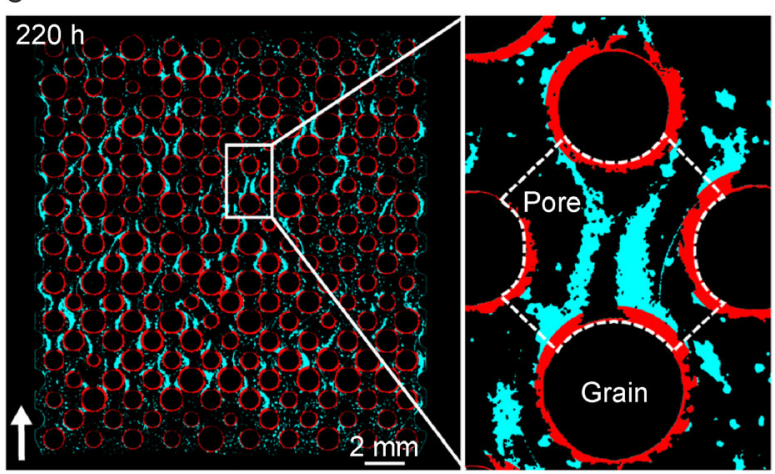

B

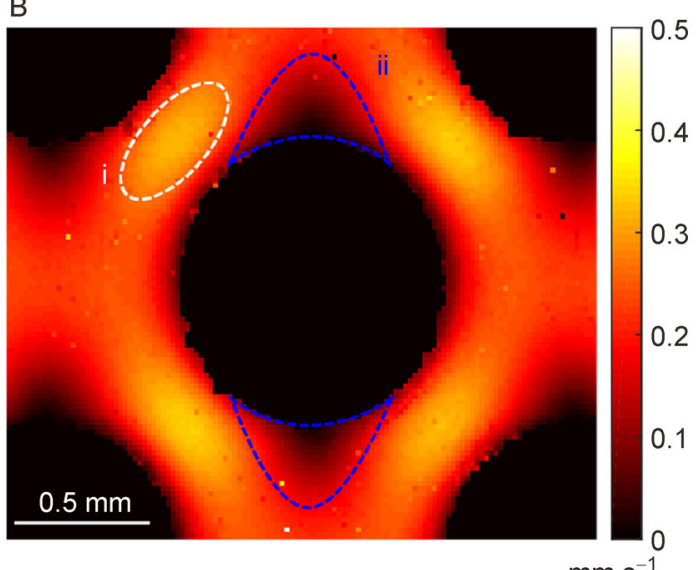

$\mathrm{mm} \mathrm{s}^{-1}$

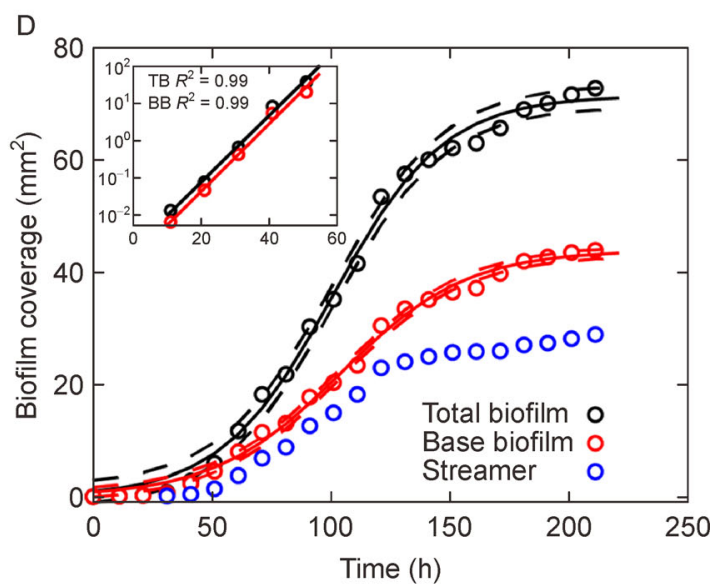

Fig. 3 The growth of multi-species biofilms in a porous environment. (A) Schematic of the microfluidic device. (B) Distribution of flow velocity in a porous environment. (C) Biofilm differentiation into streamers (cyan) and base biofilm (red) at $220 \mathrm{~h}$, which improved space utilization in porous environments. White arrow shows flow direction. (D) The growth dynamics of total biofilm (black circles), base biofilm (red circles) and streamers (blue circles) integrated in the fluidic device (Scheidweiler et al., 2019). https://doi.org/10.1038/s41396-019-0381-4

the biofilm streamers of $P$. aeruginosa PA14 in a microfluidic device and showed that biofilm streamers acted as bridges around obstacles in uneven environments, and that the internal structure of the device formed a "mesh" to capture and retain loose cells or EPS that had detached from the basal biofilm. The biofilms formed on the surfaces had little effect on flow, but the pipeline could unexpectedly be blocked by biofilm streamers, highlighting the need to investigate bacterial behavior in realistic industrial and clinical settings where biofilm prevention is critical. Kim et al. (2014) also used a continuous flow device to study biofilm streamers. Staphylococcus aureus formed biofilm streamers quickly, and when the channel surface was coated with blood plasma, a streamer would appear within minutes and quickly block the channel.

In addition, other types of microfluidic devices have been developed. For instance, Kumar et al. (2013) were inspired by shark skin to design a chip that could generate a secondary flow to study biofilms. When the flow rate exceeded $4 \mu \mathrm{L} \mathrm{h}$ (Reynolds number or $\operatorname{Re}=2 \times 10^{-3}$ ), biofilm streamers decreased with the increase of flow velocity. This chip has potential value in industrial settings as antifouling technology in the pipes. Paquet-Mercier et al. (2016) applied a microfluidic system with straight microchannels to investigate the viscosity change of $P$. fluorescens CT07 biofilms. They showed a rapid increase in biofilm viscosity, increasing by an order of magnitude in less than $10 \mathrm{~h}$. This system, which combined video tracking with a semi-empirical viscous flow model, enabled continuous measurements of intact biofilms under low unchanging laminar flow conditions and could be further used for exploring the response of biofilm viscosity under well-controlled physical, chemical, and biological growth conditions.

\subsection{Bacterial chemotaxis}

Swimming bacteria can sense chemical signals and migrate along a concentration gradient, which is known as chemotaxis. To study the chemotactic response of bacteria to organic matter in the underground environment, Singh and Olson (2012) used a microfluidic device that simulated a twodimensional dual-permeability groundwater pollution scenario. When DL-aspartic acid was used as an attractant, the 
total number of $E$. coli HCB33 increased by 1.09 to 1.74 times in the low-flow area. The chemotactic reaction decreased linearly with the increase of flow rate, and there was no chemotactic reaction at the highest flow rate (Darcy velocity = $0.22 \mathrm{~mm} / \mathrm{s}$ ). Wang et al. (2012) used a "T"-type microfluidic device to simulate chemotaxis in an underground environment. The microchip had two inlets: the narrow slanted channel was used as a side channel for nonaqueous phase liquid injection and the wide horizontal channel was the main channel for bacterial suspension. At a flow velocity of $0.5 \mathrm{~m}$ $\mathrm{d}^{-1}$, the concentration of $P$. putida F1 was $25 \%$ higher than the concentration of non-chemotropic mutant bacteria near the organic/water interface, and the amount of $E$. coli was $60 \%$ higher than the control group. Wang et al. (2015) utilized a multilayer microfluidic device to generate stable linear concentration gradients of attractants to characterize bacterial chemotaxis for model fitting and parameter evaluation. Their study yielded the chemotactic sensitivity coefficient $\chi_{0}=2 \pm 1$ $\left.\times 10^{-4} \mathrm{~cm}^{2} / \mathrm{s}\right)$ and the chemotactic receptor constant $\left(\kappa_{c}=\right.$ $0.12 \pm 0.05 \mathrm{mM})$. Wang et al. (2016) further designed a heterogeneous porous device to simulate heterogeneous features of contaminated groundwater aquifers, in which toluene was confined in a network of fine pores and $P$. putida F1 was injected through an adjacent macropore. Chemotaxis preferentially promoted bacterial migration to chemokines in low conductivity areas, resulting in the accumulation of bacteria in these spaces. However, chemotaxis was reduced when simulated groundwater flow exceeded the critical rate (5 $\mathrm{m} \mathrm{d}^{-1}$ ).

While previous studies mainly considered cell migration in response to chemoattractants, relatively few studies have focused on how the physical environment influences the collective migration of bacterial cells, including when faced with obstacles and noise. Rashid et al. (2019) used a microfluidic device containing obstacles of different physical sizes to study the response of $E$. coli to chemoattractants. The average swimming rate of $E$. coli to an attractant did not change when pillars were uniformly spaced. In the case of the chemokine gradient, E. coli changed its swimming pattern, and actively secreted a strong chemoattractant to the medium, thus enhancing migration. In the device utilized by Zhang et al. (2019), bacteria moved toward the attractant in the other end, initially gathering around the strong attractant. However, when the cell density reached a critical value, the cells formed an "escape band" and moved to the nutrient source which was chemotactically weaker, but metabolically richer. de Anna et al. (2020) designed a microfluidic device consisting of a random distribution of cylindrical obstacles with circular and crescent-shaped cross-section. The device could simulate the natural soils to captures flow disorder and chemical gradients at the pore scale to quantify the transport and dispersion of $B$. subtilis. They found that chemotaxis consumingly modulates the bacterial movement in the area of low-flow, resulting in a $100 \%$ increase in bacterial dispersion coefficient.

\subsection{Quorum sensing of bacteria}

Bacteria can secrete specific chemical signals, such as acylhomoserine lactones (AHLs) (Tecon and Or, 2017; Yang et al., 2020) to the surrounding environment. When these signal molecules reach a threshold concentration, they can stimulate the expression of specific genes that enable bacteria to better adapt to the changing environment and resist adverse conditions. This phenomenon is called bacterial quorum sensing. Microbes share their habitat with many other species who may respond to these signals as well, leading to interactions not only between members of the same species, but also interspecies interactions (Bassler and Losick, 2006; Sahari et al., 2014).

Microfluidic devices have been used to simulate different spatial structures to study QS of bacteria. Park et al. (2003) used topological structure which simulated soil and found that in a closed system starved cells sensed, and were attracted to, metabolic end products and lysate of other cells. These stressed bacteria formed solitary waves and populations that collapsed into small, enclosed structures. Burmeister et al. (2018) physically separated L-lysine-producing Corynebacterium glutamicum from an L-lysine auxotrophic mutant in a way that allowed metabolic cross-feeding. L-lysine was shown to promote the growth and development of the nutrientdeficient strain through a nano-channel.

The shape of channels and chambers can also affect the bacterial behavior. As show in Fig. 4, Cho et al. (2007) designed microfluidic devices with chambers of various shapes, and observed growth and organization of confined $E$. coli cells. It was found that the cell arrangement, growth, and collective movement direction were correlated to the shape of the confining chamber. Under the influence of QS, $E$. coli formed a highly steady-state which was more conducive to the escape of cells from the chamber, and increased access of nutrients into and evacuation of waste out of the chambers. Ribbe and Maier (2016) designed a microfluidic device composed of a main channel and multiple branches, which could capture cells at the end of each branch. This device simulated the effect of different diffusion rates (strong, medium, weak, and very weak) on QS in open systems such as the soil rhizosphere. The local capture of bacteria promoted their density-dependent differentiation in structured environments. Nadell and Bassler (2011) studied the interaction between EPS-producing and non-EPS-producing Vibrio cholerae in a simple single-chambered microfluidic device. EPS-producing bacteria regulated the production of EPS through QS and gained a significant advantage. However, EPS-producing cells were damaged when they spread to new sites. This study showed there was a basic balance between local competition and diffusion among bacteria.

Other types of microfluidic systems have also been used to study QS. Jeong et al. (2015) used a microfluidic static droplet array, which could generate a series of droplets with opposite densities of two bacteria to study QS. The system was used to 


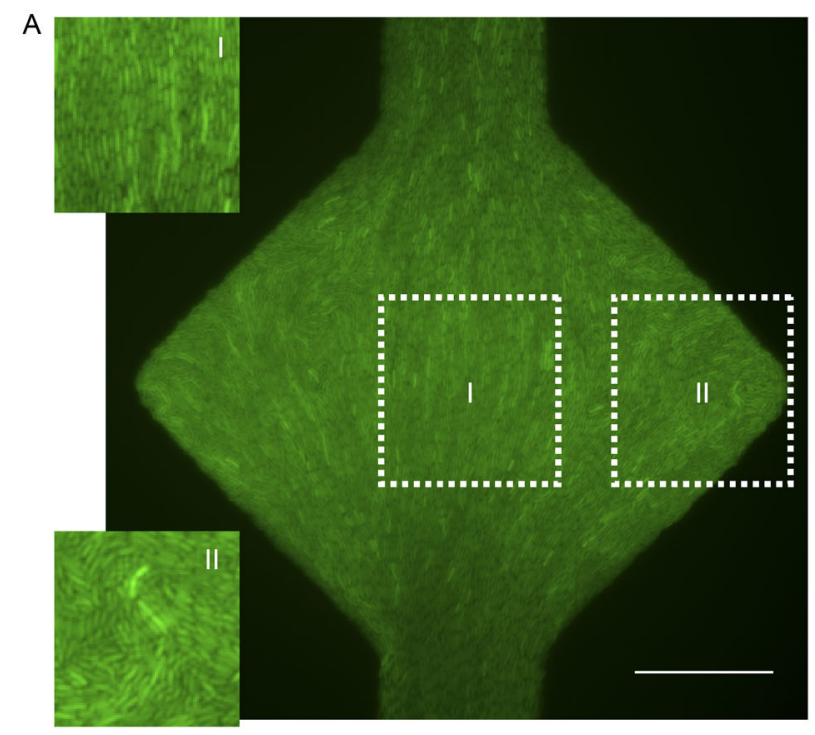

B

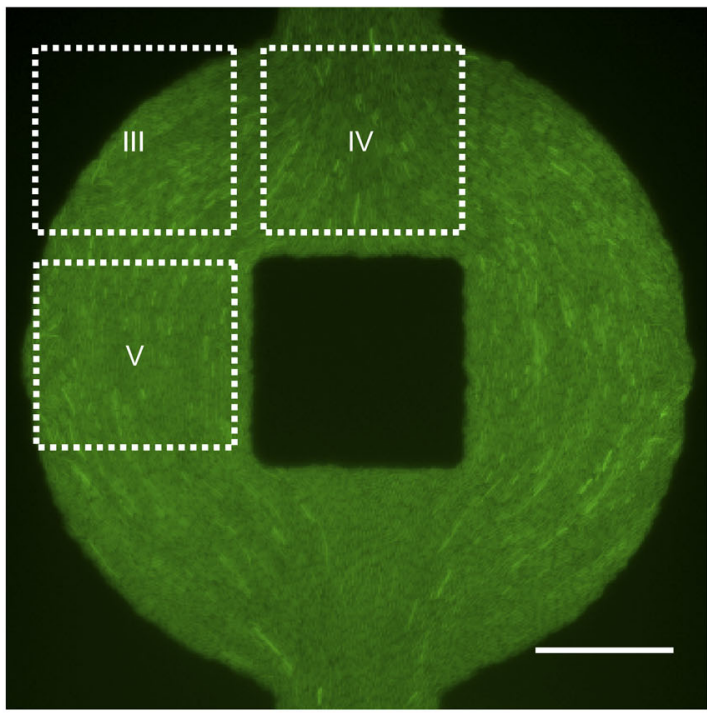

C

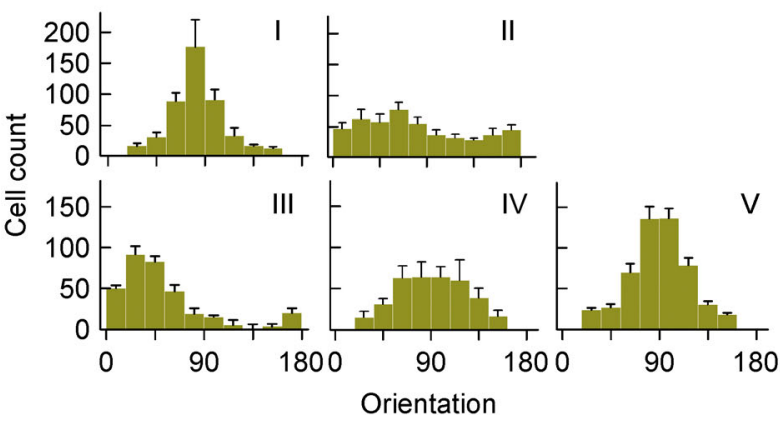

D

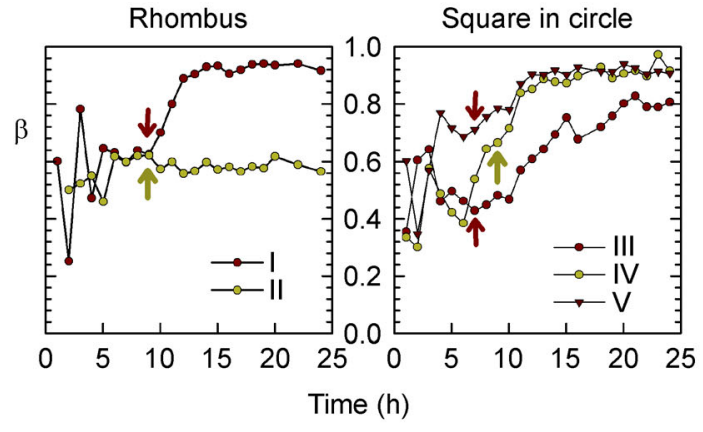

Fig. 4 Colony self-organization in microfluidic device with chambers of different shapes. Colony self-organization in (A) a rhombus and (B) a circle with square in the middle. (C) The histograms of the steady-state cell orientations in the selected regions based on horizontal direction. (D) The fraction of cells oriented within $\pm 45^{\circ}$ at the peak of the eventual steady-state histogram (Cho et al., 2007). https://doi.org/10.1371/ journal.pbio.0050302

study the effects of co-culturing AHLs-producing and AHLsreceiving bacteria at different ratios. As the proportion of signal-producing bacteria decreased and the signal receiving bacteria increased, more green fluorescent protein (GFP) was produced. Also Nagy et al. (2015) studied the interactions between bacterial QS and chemotaxis in microfluidics. Their device could quickly form a linear concentration gradient. The results indicated that signal molecules and secondary metabolites secreted by $P$. aeruginosa affected the distribution of $E$. coli. Hong et al. (2012) studied the interactions between an $E$. coli biofilm and the concentration of signal molecules using a two-layer microchip. The setup generated independent bacterial and signal molecule concentration gradients. It was observed that the amount of biofilm decreased with the decreasing signal molecule concentration. Underhill et al. (2018) explored whether Streptococcus mutans UA159 required extracellular XIP (sigX-inducing peptide) to induce comX in the ComRS group sensing system. The microfluidic device they used allowed to generate different concentration gradients after introduction of XIP $(0,600 \mathrm{nM}$ and $6 \mu \mathrm{M})$. Results showed that ComRS control of $\operatorname{com} X$ did not require
XIP in the absence of lysis. Leaman et al. (2018) studied QS of $E$. coli in a microfluidic device that was symmetric on both sides and enabled generation of concentration gradients. The QS activation time followed a power law with respect to bacterial population density. However, population structure and the gene loop noise significantly influenced this relationship.

2.5 Horizontal gene transfer of bacteria based on fluorescent labeling

Bacteria can expand their environmental niche by HGT, acquiring genes which are beneficial for their survival and reproduction. Under strong selection conditions (e.g., heavy metals or antibiotics), horizontally transferred genes will be quickly fixed within a bacterial group (Heuer and Smalla, 2012; Tecon and Or, 2017; Wu et al., 2020). Microfluidics devices used with appropriate fluorescent labels can provide unique insights into HGT in natural environments. Qiu et al. (2018) simulated an open environment biofilm and used GFPtagged plasmids to quantify the plasmid transfer frequency. 
The results indicated that the conjugative potential of Gramnegative bacteria was higher, with the phyla Proteobacteria and Firmicutes more prone to HGT than Bacteroidetes. Li et al. (2019) also used a microfluidic device and a fluorescent labeling technique similar to Qiu et al. (2018) to study the ability of bacteria to spread antibiotic resistance genes. They quantified the transfer frequency between $E$. coli and bacteria from activated sludge and found the transfer frequencies were between $1 \times 10^{-3}-4.3 \times 10^{-2}$ per recipient. HGT mainly occurred in Gram-negative bacteria, which is consistent with the results of Qiu et al. (2018). Labeling with fluorescent proteins has also enabled to demonstrate that direct contact between bacteria is the key to gene transfer. For example, Zhu et al. (2019) used a microfluidic device to coculture E. coli with recipient bacteria isolated from activated sludge. HGT occurred at the early stage, and the frequency increased as the possibility of contact between bacteria rose after emergence of the community. Cooper et al. (2018) designed a monolayer device to coculture bacteria and found that the plasmid pBAV1k-GFP of $E$. coli transferred to Acinetobacter baylyi after they came in contact with each other. Burmeister et al. (2018) found that $P$. putida was not able to produce yellow fluorescence when bacteria were cocultured in a space-separated device, while contact between the cells caused a change in the fluorescent signal from red to yellow, indicating the occurrence of HGT. Pivetal et al. (2015) designed a new microfluidic system composed of micromagnets to monitor HGT. Magnetic nanoparticles were used to label plasmid DNA molecules and magnetic microfluidic devices were then employed to capture and separate the recombinant cells with nanoparticle signals. The study provided a novel alternative method for studying the extent of HGT occurring in the natural environments.

\section{Outlook}

The introduction of microfluidics has significantly advanced the opportunities for dynamic visualization and analysis of bacteria, which greatly promotes future research in a range of areas including the morphology and function of soil microorganisms, soil biofilm formation, bacterial chemotaxis, QS, and HGT. It also brings new opportunities for microbiology by simulating soil structure to study the effects of microbial EPS on soil water retention and bacterial distribution, the hydrodynamic mechanisms of biofilm streamers, and the rapid detection of environmental microorganisms. These research topics can help us better understand the nature of bacteria, and the ways they are engaged in a variety of biochemical reactions with other bacteria and the surrounding environment. The study of these interactions is essential for understanding and regulating bacterial behaviors.

However, the application of microfluidics in microbial research faces several challenges. (1) The habitat of bacteria is often extremely complex, in both spatial structure and chemical conditions (e.g., soil). Current microfluidic devices can only simulate relatively simple soil structures. Therefore, microchips that are closer to natural bacterial habitats need to be developed to better reveal the interactions between bacteria and their environment. (2) Bacterial screening, including the screening of unknown microorganisms in the environment and "core microorganisms" in the rhizosphere, is one developing trend in microfluidics. Core microbial communities can "recruit" microorganisms which are beneficial for the plant growth and can effectively inhibit the growth of pathogens. Research in this area is still limited. So far, microfluidic technology has been utilized mainly for studying isolated and cultured bacteria, but there are many barriers to the isolation and culture of the remaining $95 \%$. Therefore, the development of micro-droplet and other microfluidic devices will be beneficial in accelerating the separation, cultivation, and screening of these organisms. (3) To date, most microfluidics studies have focused on the mechanisms of bacteria, but only a few studies have explored microfluidic devices for applications, such as environmental diagnostic systems. To overcome these challenges, it is necessary to increase the connections between microfluidics and other technologies, including not only microscopy, spectroscopy, and electrochemistry, but also the soft photolithography and etching technology for device fabrication. The integration of multiple technologies is expected to become the defining trend of microfluidics.

\section{Acknowledgments}

This work was supported by the National Natural Science Foundation of China (41877029, 41961130383), Royal SocietyNewton Advanced Fellowship (NAFIR1\191017), and Wuhan Science and Technology Bureau (2019020701011469). No potential conflicts of interest were reported by the authors.

\section{References}

Aleklett, K., Kiers, E.T., Ohlsson, P., Shimizu, T.S., Caldas, V.E., Hammer, E.C., 2018. Build your own soil: exploring microfluidics to create microbial habitat structures. ISME Journal 12, 312-319.

Aufrecht, J.A., Fowlkes, J.D., Bible, A.N., Morrell-Falvey, J., Doktycz, M.J., Retterer, S.T., 2019. Pore-scale hydrodynamics influence the spatial evolution of bacterial biofilms in a microfluidic porous network. PLoS One 14, e0218316.

Bassler, B.L., Losick, R., 2006. Bacterially speaking. Cell 125, 237 246.

Biswas, I., Ghosh, R., Sadrzadeh, M., Kumar, A., 2018. Near wall void growth leads to disintegration of colloidal bacterial streamer. Journal of Colloid and Interface Science 522, 249-255.

Borer, B., Tecon, R., Or, D., 2018. Spatial organization of bacterial populations in response to oxygen and carbon counter-gradients in pore networks. Nature Communications 9, 769.

Burmeister, A., Hilgers, F., Langner, A., Westerwalbesloh, C., Kerkhoff, Y., Tenhaef, N., Drepper, T., Kohlheyer, D., von Lieres, E., Noack, S., Grünberger, A., 2018. A microfluidic co-cultivation platform to investigate microbial interactions at defined micro- 
environments. Lab on a Chip 19, 98-110.

Cai, P., Sun, X.J., Wu, Y.C., Gao, C.H., Mortimer, M., Holden, P.A., Redmile-Gordon, M., Huang, Q.Y., 2019. Soil biofilms: microbial interactions, challenges, and advanced techniques for ex-situ characterization. Soil Ecology Letters 1, 85-93.

Cho, H., Jönsson, H., Campbell, K., Melke, P., Williams, J.W. Jedynak, B., Stevens, A.M., Groisman, A., Levchenko, A., 2007. Self-organization in high-density bacterial colonies: efficient crowd control. PLoS Biology 5, e302.

Cooper, R., Tsimring, L., Hasty, J., 2018. Microfluidics-based analysis of contact-dependent bacterial interactions. Bio-Protocol 8, 8.

Coyte, K.Z., Tabuteau, H., Gaffney, E.A., Foster, K.R., Durham, W.M., 2017. Microbial competition in porous environments can select against rapid biofilm growth. Proceedings of the National Academy of Sciences of the United States of America 114, E161-E170.

de Anna, P., Pahlavan, A.A., Yawata, Y., Stocker, R., Juanes, R., 2020. Chemotaxis under flow disorder shapes microbial dispersion in porous media. Nature Physics. https://doi.org/10.1038/s41567020-1002-x

Deng, J., Orner, E.P., Chau, J.F., Anderson, E.M., Kadilak, A.L., Rubinstein, R.L., Bouchillon, G.M., Goodwin, R.A., Gage, D.J., Shor, L.M., 2015. Synergistic effects of soil microstructure and bacterial EPS on drying rate in emulated soil micromodels. Soil Biology \& Biochemistry 83, 116-124.

Drescher, K., Shen, Y., Bassler, B.L., Stone, H.A., 2013. Biofilm streamers cause catastrophic disruption of flow with consequences for environmental and medical systems. Proceedings of the National Academy of Sciences of the United States of America 110, 4345-4350.

Feng, J., de la Fuente-Núñez, C., Trimble, M.J., Xu, J., Hancock, R.E. W., Lu, X., 2015. An in situ Raman spectroscopy-based microfluidic "lab-on-a-chip" platform for non-destructive and continuous characterization of Pseudomonas aeruginosa biofilms. Chemical Communications 51, 8966-8969.

Flemming, H.C., Wuertz, S., 2019. Bacteria and archaea on Earth and their abundance in biofilms. Nature Reviews. Microbiology 17 , 247-260.

Guo, Y.S., Furrer, J.M., Kadilak, A.L., Hinestroza, H.F., Gage, D.J., Cho, Y.K., Shor, L.M., 2018. Bacterial extracellular polymeric substances amplify water content variability at the pore scale. Frontiers in Environmental Science 6, 93.

Gutiérrez Castorena, E.V., Gutiérrez-Castorena, M., González Vargas, T., Cajuste Bontemps, L., Delgadillo Martínez, J., Suástegui Méndez, E., Ortiz Solorio, C.A., 2016. Micromapping of microbial hotspots and biofilms from different crops using digital image mosaics of soil thin sections. Geoderma 279, 11-21.

Hassanpourfard, M., Ghosh, R., Thundat, T., Kumar, A., 2016. Dynamics of bacterial streamers induced clogging in microfluidic devices. Lab on a Chip 16, 4091-4096.

Hassanpourfard, M., Sun, X., Valiei, A., Mukherjee, P., Thundat, T., Liu, Y., Kumar, A., 2014. Protocol for biofilm streamer formation in a microfluidic device with micropillars. Jove-Journal of Visualized Experiments 90, e51732.

Heuer, H., Smalla, K., 2012. Plasmids foster diversification and adaptation of bacterial populations in soil. FEMS Microbiology Reviews 36, 1083-1104.
Hong, S.H., Hegde, M., Kim, J., Wang, X., Jayaraman, A., Wood, T.K., 2012. Synthetic quorum-sensing circuit to control consortial biofilm formation and dispersal in a microfluidic device. Nature Communications 3, 613.

Huang, X.Z., Li, Y.W., Guggenberger, G., Kuzyakov, Y., Liu, B.F., Wu, J.S., 2020. Direct evidence for thickening nanoscale organic films at soil biogeochemical interfaces and its relevance to organic matter preservation. Environmental Science: Nano 7, 2747-2758.

Huang, X.Z., Li, Y.W., Liu, B.F., Guggenberger, G., Shibistova, O., Zhu, Z.K., Ge, T.D., Tan, W.F., Wu, J.S., 2017. SoilChip-XPS integrated technique to study formation of soil biogeochemical interfaces. Soil Biology \& Biochemistry 113, 71-79.

Jeong, H.H., Jin, S.H., Lee, B.J., Kim, T., Lee, C.S., 2015. Microfluidic static droplet array for analyzing microbial communication on a population gradient. Lab on a Chip 15, 889-899.

Karimi, A., Karig, D., Kumar, A., Ardekani, A.M., 2015. Interplay of physical mechanisms and biofilm processes: review of microfluidic methods. Lab on a Chip 15, $23-42$.

Kim, J.W., Choi, H., Pachepsky, Y.A., 2010. Biofilm morphology as related to the porous media clogging. Water Research 44, 11931201.

Kim, M.K., Drescher, K., Pak, O.S., Bassler, B.L., Stone, H.A., 2014. Filaments in curved streamlines: Rapid formation of Staphylococcus aureus biofilm streamers. New Journal of Physics 16, 065024.

Koffler, H., Knight, S.G., Emerson, R.L., Burris, R.H., 1945. The effect of certain chemicals on penicillin production and mold metabolism in shake flask fermentations. Journal of Bacteriology 50, 549-559.

Kumar, A., Karig, D., Acharya, R., Neethirajan, S., Mukherjee, P.P., Retterer, S., Doktycz, M.J., 2013. Microscale confinement features can affect biofilm formation. Microfluidics and Nanofluidics 14 , 895-902.

Leaman, E.J., Geuther, B.Q., Behkam, B., 2018. Quantitative investigation of the role of intra-/intercellular dynamics in bacterial quorum sensing. ACS Synthetic Biology 7, 1030-1042.

Li, B., Qiu, Y., Zhang, J., Liang, P., Huang, X., 2019. Conjugative potential of antibiotic resistance plasmids to activated sludge bacteria from wastewater treatment plants. International Biodeterioration \& Biodegradation 138, 33-40.

Männik, J., Driessen, R., Galajda, P., Keymer, J.E., Dekker, C., 2009. Bacterial growth and motility in sub-micron constrictions. Proceedings of the National Academy of Sciences of the United States of America 106, 14861-14866.

Marty, A., Roques, C., Causserand, C., Bacchin, P., 2012. Formation of bacterial streamers during filtration in microfluidic systems. Biofouling 28, 551-562.

Miyake, Y., Fujiwara, S., Usui, T., Suginaka, H., 1992. Simple method for measuring the antibiotic concentration required to kill adherent bacteria. Chemotherapy 38, 286-290.

Mukherjee, M., Menon, N.V., Liu, X., Kang, Y., Cao, B., 2016. Confocal laser scanning microscopy-compatible microfluidic membrane flow cell as a nondestructive tool for studying biofouling dynamics on forward osmosis membranes. Environmental Science \& Technology Letters 3, 303-309.

Nadell, C.D., Bassler, B.L., 2011. A fitness trade-off between local competition and dispersal in Vibrio cholerae biofilms. Proceedings of the National Academy of Sciences of the United States of 
America 108, 14181-14185.

Nagy, K., Sipos, O., Valkai, S., Gombai, É., Hodula, O., Kerényi, Á., Ormos, P., Galajda, P., 2015. Microfluidic study of the chemotactic response of Escherichia coli to amino acids, signaling molecules and secondary metabolites. Biomicrofluidics 9, 044105.

Nunan, N., Wu, K., Young, I.M., Crawford, J.W., Ritz, K., 2003. Spatial distribution of bacterial communities and their relationships with the micro-architecture of soil. FEMS Microbiology Ecology 44, 203-215.

Paquet-Mercier, F., Parvinzadeh Gashti, M., Bellavance, J., Taghavi, S.M., Greener, J., 2016. Through thick and thin: a microfluidic approach for continuous measurements of biofilm viscosity and the effect of ionic strength. Lab on a Chip 16, 4710-4717.

Park, S., Wolanin, P.M., Yuzbashyan, E.A., Lin, H., Darnton, N.C., Stock, J.B., Silberzan, P., Austin, R., 2003. Influence of topology on bacterial social interaction. Proceedings of the National Academy of Sciences of the United States of America 100, 13910-13915.

Pivetal, J., Frénéa-Robin, M., Haddour, N., Vézy, C., Zanini, L.F., Ciuta, G., Dempsey, N.M., Dumas-Bouchiat, F., Reyne, G., BéginColin, S., Felder-Flesh, D., Ghobril, C., Pourroy, G., Simonet, P., 2015. Development and applications of a DNA labeling method with magnetic nanoparticles to study the role of horizontal gene transfer events between bacteria in soil pollutant bioremediation processes. Environmental Science and Pollution Research International 22, 20322-20327.

Pousti, M., Zarabadi, M.P., Abbaszadeh Amirdehi, M., PaquetMercier, F., Greener, J., 2018. Microfluidic bioanalytical flow cells for biofilm studies: a review. Analyst (London) 144, 68-86.

Qiu, Y., Zhang, J., Li, B., Wen, X., Liang, P., Huang, X., 2018. A novel microfluidic system enables visualization and analysis of antibiotic resistance gene transfer to activated sludge bacteria in biofilm. Science of the Total Environment 642, 582-590.

Rashid, S., Long, Z., Singh, S., Kohram, M., Vashistha, H., Navlakha, S., Salman, H., Oltvai, Z.N., Bar-Joseph, Z., 2019. Adjustment in tumbling rates improves bacterial chemotaxis on obstacle-laden terrains. Proceedings of the National Academy of Sciences of the United States of America 116, 11770-11775.

Ribbe, J., Maier, B., 2016. Density-dependent differentiation of bacteria in spatially structured open systems. Biophysical Journal 110, 1648-1660.

Roberson, E.B., Firestone, M.K., 1992. Relationship between dessication and exopolysaccharide production in a soil Pseudomonas sp. Applied and Environmental Microbiology 58, 1284 1291.

Sahari, A., Traore, M.A., Stevens, A.M., Scharf, B.E., Behkam, B., 2014. Toward development of an autonomous network of bacteriabased delivery systems (BacteriaBots): spatiotemporally highthroughput characterization of bacterial quorum-sensing response. Analytical Chemistry 86, 11489-11493.

Salek, M.M., Carrara, F., Fernandez, V., Guasto, J.S., Stocker, R., 2019. Bacterial chemotaxis in a microfluidic T-maze reveals strong phenotypic heterogeneity in chemotactic sensitivity. Nature Communications 10, 1877.

Scheidweiler, D., Peter, H., Pramateftaki, P., de Anna, P., Battin, T.J., 2019. Unraveling the biophysical underpinnings to the success of multispecies biofilms in porous environments. ISME Journal 13, $1700-1710$.

Singh, A.K., Prakash, P., Singh, R., Nandy, N., Firdaus, Z., Bansal, M., Singh, R.K., Srivastava, A., Roy, J.K., Mishra, B., Singh, R.K., 2017. Curcumin quantum dots mediated degradation of bacterial biofilms. Frontiers in Microbiology8, 1517.

Singh, R., Olson, M.S., 2012. Transverse chemotactic migration of bacteria from high to low permeability regions in a dual permeability microfluidic device. Environmental Science \& Technology 46, 3188-3195.

Stewart, E.J., 2012. Growing unculturable bacteria. Journal of Bacteriology 194, 4151-4160.

Tecon, R., Or, D., 2017. Biophysical processes supporting the diversity of microbial life in soil. FEMS Microbiology Reviews 41, 599-623.

Toju, H., Peay, K.G., Yamamichi, M., Narisawa, K., Hiruma, K., Naito, K., Fukuda, S., Ushio, M., Nakaoka, S., Onoda, Y., Yoshida, K., Schlaeppi, K., Bai, Y., Sugiura, R., Ichihashi, Y., Minamisawa, K., Kiers, E.T., 2018. Core microbiomes for sustainable agroecosystems. Nature Plants 4, 247-257.

Underhill, S.A.M., Shields, R.C., Kaspar, J.R., Haider, M., Burne, R.A., Hagen, S.J., 2018. Intracellular signaling by the comRS system in Streptococcus mutans genetic competence. MSphere 3, e00444e18.

Valiei, A., Kumar, A., Mukherjee, P.P., Liu, Y., Thundat, T., 2012. A web of streamers: biofilm formation in a porous microfluidic device. Lab on a Chip 12, 5133-5137.

Wang, X., Atencia, J., Ford, R.M., 2015. Quantitative analysis of chemotaxis towards toluene by Pseudomonas putida in a convection-free microfluidic device. Biotechnology and Bioengineering 112, 896-904.

Wang, X., Lanning, L.M., Ford, R.M., 2016. Enhanced retention of chemotactic bacteria in a pore network with residual NAPL contamination. Environmental Science \& Technology 50, 165-172.

Wang, X., Long, T., Ford, R.M., 2012. Bacterial chemotaxis toward a NAPL source within a pore-scale microfluidic chamber. Biotechnology and Bioengineering 109, 1622-1628.

Weaver, W.M., Milisavljevic, V., Miller, J.F., Di Carlo, D., 2012. Fluid flow induces biofilm formation in Staphylococcus epidermidis polysaccharide intracellular adhesin-positive clinical isolates. Applied and Environmental Microbiology 78, 5890-5896.

Wu, S., Wu, Y.C., Huang, Q.Y., Cai, P., 2020. Insights into conjugative transfer of antibiotic resistance genes affected by soil minerals. European Journal of Soil Science.

Wu, X., Staggenborg, S., Propheter, J.L., Rooney, W.L., Yu, J., Wang, D., 2010. Features of sweet sorghum juice and their performance in ethanol fermentation. Industrial Crops and Products 31, 164 170.

Wu, Y., Cai, P., Jing, X., Niu, X., Ji, D., Ashry, N.M., Gao, C., Huang, Q., 2019. Soil biofilm formation enhances microbial community diversity and metabolic activity. Environment International 132, 105116

Yang, S.S., Qu, C.C., Mukherjee, M., Wu, Y.C., Huang, Q.Y., Cai, P., 2020. Soil phyllosilicate and iron oxide inhibit the quorum sensing of Chromobacterium violaceum. Soil Ecology Letters. https://doi. org/10.1007/s42832-020-0051-5. 
Yazdi, S., Ardekani, A.M., 2012. Bacterial aggregation and biofilm formation in a vortical flow. Biomicrofluidics 6, 44114.

Zambare, N.M., Lauchnor, E.G., Gerlach, R., 2019. Controlling the distribution of microbially precipitated calcium carbonate in radial flow environments. Environmental Science \& Technology 53, 5916-5925.

Zhang, X., Si, G., Dong, Y., Chen, K., Ouyang, Q., Luo, C., Tu, Y., 2019. Escape band in Escherichia coli chemotaxis in opposing attractant and nutrient gradients. Proceedings of the National
Academy of Sciences of the United States of America 116, 22532258.

Zhu, Y., Qiu, Y., Li, B., Wang, S., Li, J., 2019. Transfer dynamics of antibiotic resistance genes in sludge bacterial community using live cell 3D imaging technology. Acta Scientiae Circumstantiae 39, 2218-2223.

Zhu, Y.G., Shen, R.F., He, J.Z., Wang, Y.F., Han, X.G., Jia, Z.J., 2017. China soil microbiome initiative: progress and perspective. Bulletin of the Chinese Academy of Sciences 32, 554-565. 